\title{
EDITORIAL \}
}

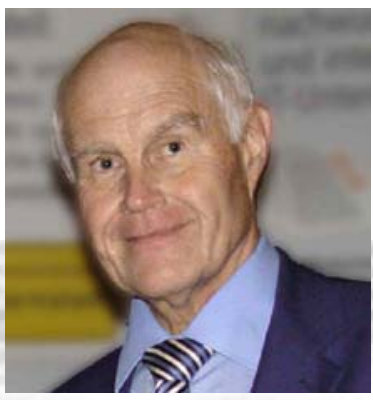

Prof. Dr. Peter Mertens Universität Erlangen-Nürnberg

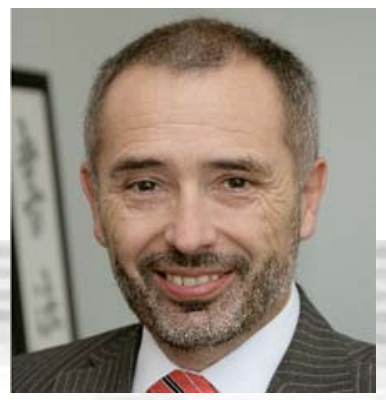

Prof. Dr. Dieter Steinbauer SCHUFA Holding AG

\section{Finanzkrise und IT}

Die IT hat die neueren globalen Bankgeschäfte hinsichtlich Datenversorgung, Rechenvorgängen und Geschwindigkeit der Datenübertragung erst ermöglicht und gilt daher als „mitschuldig" an der Finanzkrise. Zu Recht erwartet man nun von der Informatik und von der Wirtschaftsinformatik einen Beitrag zur Krisenbewältigung und zur Vorbeugung. Dem tragen die Beiträge in diesem Themenheft Rechnung. Freilich darf eine geschlossenen Darstellung mit nur drei Hauptbeiträgen nicht erwartet werden.

Müller u. a. weisen darauf hin, dass bei den vielfältigen Bemühungen um die Krisenvorbeugung und -bewältigung die IT-Infrastruktur stark vernachlässigt worden ist. Das überrascht, ist sie doch Träger und Werkzeug des weltweiten Handels mit Finanzprodukten. In ihrem Beitrag diskutieren die Autoren Eigenschaften der zur ITInfrastruktur gehörenden Sicherheitsmodelle anhand eines Vier-Stufen-Schemas. Ein besonderes Anliegen ist, beim Aufbau eines Regelwerks der Nutzung der IT-Infrastruktur im Vergleich zur reinen Zugangskontrolle ein höheres Gewicht zu verleihen, denn die bisherige Vernachlässigung der Nutzungsregeln ist eine Ursache für die mangelnde Transparenz der Finanzmärkte.

Mertens geht von der bei der Finanzkrise vielfach sichtbar gewordenen, ungenügenden Überwachung der Vorstände durch Kontrollorgane, z. B. Aufsichtsräte oder Verwaltungsräte, aus. Er plädiert daher für weitgehend automatisierte Überwachungssysteme, wie sie in anderen risikobehafteten Bereichen (Kernkraftwerke, Flugbetrieb) schon lange üblich sind, so dass Angestellte bis hinauf zum Vorstand, die Berichte an die Kontrollinstanzen manipulieren könnten, keine Möglichkeit dazu haben. Er zeigt, wie sich eine ganze Reihe von Instrumenten der Informatik/Wirtschaftsinformatik (unter anderem Modellierung von hochstrukturierten Wertpapieren und Kreditverbriefungen unter Benutzung von Methoden der Grunddatenverwaltung im Fertigungssektor (Stücklisten, Teileverwendungsstammsätze), Data Mining, Expertisesysteme zur automatischen Analyse und Kommentierung von Zahlenwerken, Computergrafik zur Visualisierung, Frühwarnsysteme) zu einem geschlossenen Informationssystem für Kontrollgremien verbinden lassen.

Das Anliegen von Hamerle/Plank ist es, Informationsdefizite und -asymmetrien zwischen Marktteilnehmern, die bei strukturierten Produkten wie ABS oder CDO eine besondere Hebelwirkung entfalten, zu analysieren und die erhöhte Sensitivität der Ratings auf fehlerhafte Eingangsdaten oder umfangreiche Fehlkalibrierungen zurückzuführen. Die Autoren bedienen sich umfangreicher Simulationen, um den Einfluss zahlreicher Parameter aufzuhellen. Hamerle und Plank belegen sehr deutlich, dass Informatik/Wirtschaftsinformatik zahlreiche Hilfen stellen können, z. B. bei den benötigten Datenmodellen, bei automatischen Rating- und Bewertungssystemen, bei der Schaffung von öffentlichen Schnittstellen und bei der Übertragung von Kenntnissen aus anderen Netzwerkindustrien auf Bankgeschäfte.

\section{Prof. Dr. Peter Mertens, Prof. Dr. Dieter Steinbauer}

